\title{
Our Experience with Supratracheal Partial Laryngectomy in Advanced Laryngeal Cancer: A Report of Five Cases
}

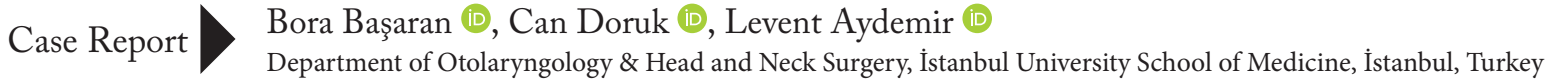

Abstract

ORCID iDs of the authors: B.B. 0000-0003-0251-5895; C.D. 0000-0002-4983-3666; L.A. 0000-0002-5836-4304.

Cite this article as: Başaran B, DorukC, Aydemir L. Our Experience with Supratracheal Partial Laryngectomy in Advanced Laryngeal Cancer: A Report of Five Cases. Turk Arch Otorhinolaryngol 2020; 58(3): 193-6.

\section{Corresponding Author:}

Can Doruk, can.doruk@istanbul.edu.tr

Received Date: 06.01.2020

Accepted Date: 12.05 .2020

Content of this journal is licensed under a Creative Commons Attribution 4.0 International License. Available online at www.turkarchotolaryngol.net
Supratracheal laryngectomy (STL) is the ultimate option in conservation surgery, and can be used as an alternative to total laryngectomy in selected tumors. This case series is the first report from Turkey that analyzes the oncological and functional outcomes of STL along with a literature review. Files of five patients who underwent STL between June 2016 and June 2019 were analyzed. Primary tumor findings, postoperative pathology reports, treatment modalities and oncological and functional outcomes of the surgical intervention were noted. Two patients were operated on for horse-shoe glottic tumors with subglottic extension, one patient for primary subglottic mass and two for transglottic ventricular tumors with an- terior subglottic extension. Pathological stage of two patients was T3, and of three patients was T4a due to thyroid cartilage invasion. No neck metastases were observed. Three patients with T4a primary tumor were treated with adjuvant radiotherapy. All patients were found disease free in their last follow-up visit. Nasogastric tube was removed in the $1^{\text {st }}$ postoperative month in all patients. While one patient remained tracheostomized in the follow-up period, the remaining four were decannulated. This technique can be used as an alternative to total laryngectomy especially in selected cases with subglottic extension.

Keywords: Larynx cancer, conservation surgery, laryngectomy, supratracheal laryngectomy

\section{Introduction}

One of the most common techniques used in open conservation surgery for advanced larynx cancer is supracricoid laryngectomy (SCL) described by Majer and Rieder (1) in 1959 and popularized by Laccourreye et al. (2). The first modification of SCL, which was the first approach to an extended supracricoid laryngectomy, was described by Laccourreye et al. (3) in 1996. The referred study described the resection of the cricoid cartilage for glottic tumors with anterior subglottic extension. The first "functional" supratracheal laryngectomy (STL) was described by Rizzotto et al. (4) in 2006 as a modification of SCL with the aim of wider resection margins. After its oncologic and functional reliability was demonstrated, this technique was included in the new open partial horizontal laryngectomy (OPHL) classification by the European Laryngology Society in 2014 (5). In this new classification system, OPHLs are classified into three groups based on the craniocaudal extension of the resection: type 1 - supraglottic laryngectomy, type 2 - supracricoid laryngectomy, and type 3 - supratracheal laryngectomy (5).

STL is the ultimate option of the OPHLs with the widest resection limits, and it can be used in selected tumors (such as those with subglottic extension) for which the SCL method is not suitable. The purpose of STL is to provide a wide laryngeal resection while avoiding permanent tracheostomy and by preserving the phonation, protection and respiration functions.

This case series report is the first from Turkey to analyze the oncological and functional outcomes of STL along with a literature review.

\section{Case Presentation}

Written informed consents were taken from all patients. All patients included to the study were operated for squamous cell carcinoma of the larynx. 


\section{Case 1}

A 43-year-old male patient underwent STL and anterior neck dissection (level VI) for a horse-shoe glottic tumor with anterior subglottic extension (Figure 1). Postoperative histopathologic examination revealed the presence of thyroid cartilage invasion with no lymph node metastasis and with clear margins. The patient received adjuvant radiotherapy as the tumor was stage T4a.- The patient was decannulated in the $3^{\text {rd }}$ week and the nasogastric tube was removed in the $22^{\text {nd }}$ week. In the $52^{\text {nd }}-$ month follow-up, the patient was found radiologically and clinically disease-free. His Voice Related Quality of Life (VRQoL) score was 25 and Eating Assessment Tool (EAT-10) score was 10.

\section{Case 2}

A 53-year-old male patient underwent STL for a right balanced subglottic ulcerative-vegetative mass. Additionally, anterior and right-sided paratracheal neck dissections (level VI) with right thyroid lobo-isthmectomy were performed. Even though the surgical margins were clear and no lymph node metastasis was present, full-thickness thyroid cartilage invasion was reported. The patient received adjuvant radiotherapy as the tumor was stage T4a. The nasogastric tube was removed in the $3^{\text {rd }}$ week and the patient was decannulated in the $10^{\text {th }}$ week, following the completion of radiotherapy, after the surgery. No recurrence was observed in endoscopic examination at the $8^{\text {th }}$-month follow-up. The VRQoL score of the patient was 19 and the EAT-10 score was 14 .

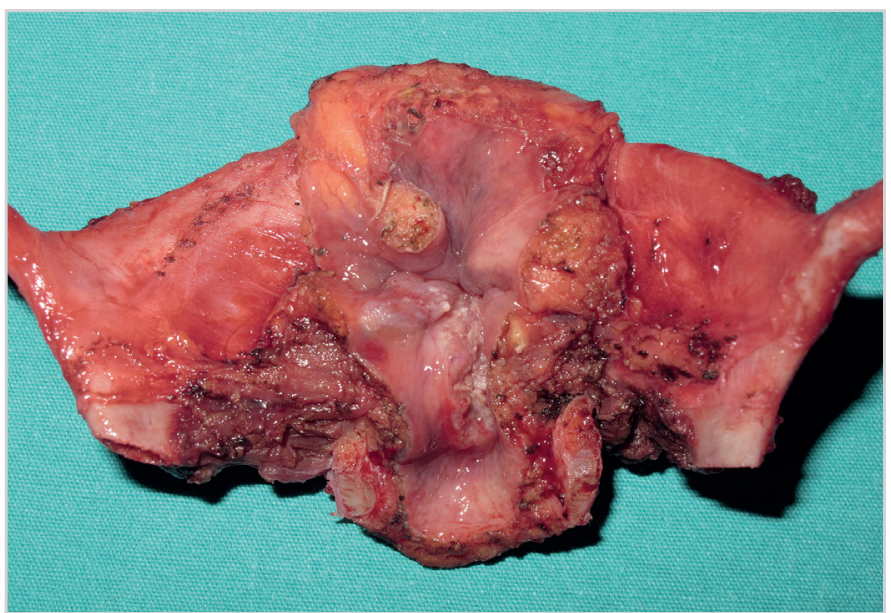

Figure 1. Horse-shoe glottic tumor with mucosal subglottic extension

\section{Main Points}

- Supratracheal partial laryngectomy is an oncologically safe procedure for selected cases with advanced glottic laryngeal cancer.

- At least one cricoarytenoid unit must be preserved in order to maintain laryngeal functions properly after supratracheal partial laryngectomy.

- Even though the vocal functions are affected after the surgery, the phonatory functions of patients who underwent supratracheal partial laryngectomy are similar to patients who underwent supracricoid partial laryngectomy.

- Most of the patients can start oral feeding without any extra supplement within the first month after surgery.

\section{Case 3}

A 61-year-old male patient underwent STL with anterior (level VI) and right-sided (level IIa-b, III and IV) neck dissections for a right ventricle tumor extending to the subglottis and the anterior commissure (Figure 2). The right vocal fold was immobile, but the crico-arytenoid joint was intact. Even though the cricoarytenoid unit was not involved, the ipsilateral arytenoid cartilage was resected for to successfully remove the entire paraglottic space. Histopathological examination of the specimen showed clear surgical margins and no neck metastasis, but full-thickness thyroid cartilage invasion. The patient received adjuvant radiotherapy as the tumor was stage T4a. The patient was found disease-free at the $10^{\text {th }}$-month follow-up and endoscopic examination. The patient had normal oral intake with no complications or extra supplementation, and his EAT-10 score was 15 . However, the patient was still not decannulated by the $10^{\text {th }}$-month due to edema and related inadequate airway passage secondary to radiotherapy.

\section{Case 4}

A 63-year-old male patient underwent STL with anterior (level VI) and left-sided (level IIa-b, III and IV) neck dissection for a left ventricle tumor invading the left vocal fold and the ventricular band. The tumor extended to the subglottis anteriorly and the arytenoid cartilage posteriorly without cartilage invasion. Even though the cricoarytenoid unit was not involved, the ipsilateral arytenoid cartilage was resected for to successfully remove the entire paraglottic space. The postoperative histopathological examination revealed a T3 tumor stage with no neck metastasis and with clear margins; therefore, no adjuvant therapy was given. The patient was decannulated and the nasogastric tube was removed in the $4^{\text {th }}$ week after surgery. In the $39^{\text {th }}$ month follow-up visit, the patient was found disease-free both endoscopically and radiologically. His VRQoL score was 33 and EAT-10 score was 19.

\section{Case 5}

A 59-year-old male patient underwent STL and anterior neck dissection (level VI) for a horse-shoe glottic tumor with subglottic extension. Postoperative histopathologic examination showed a T3 tumor stage with no neck metastasis; therefore, no adjuvant therapy was given. The patient was decannulated

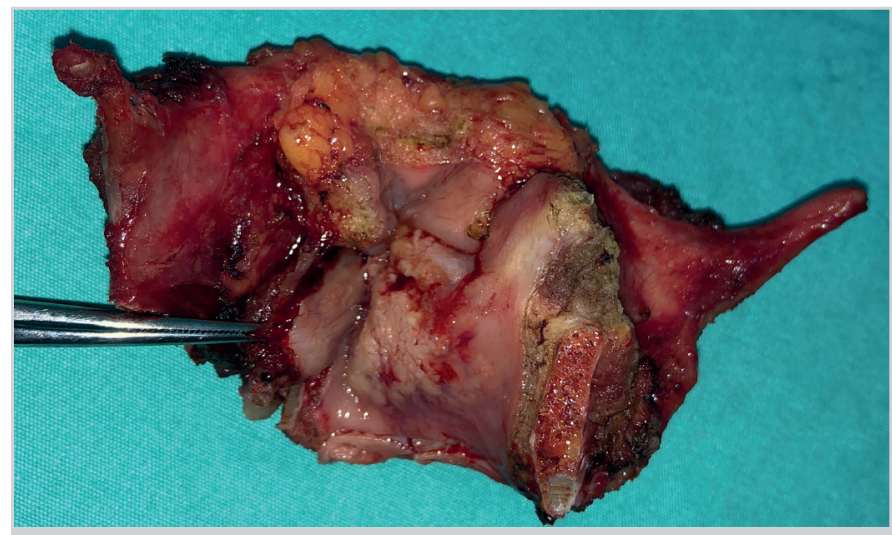

Figure 2. Transglottic ventricular tumor with anterior subglottic extension 
and the nasogastric tube was removed in the $3^{\text {rd }}$ week after the surgery. At the $16^{\text {th }}$-month follow-up, the patient was found disease-free both endoscopically and radiologically. His VRQoL score was 15 and EAT-10 score was 11.

\section{Discussion}

STL is similar to that of SCL; the basis of the larynx consists of cricoid and arytenoid cartilages, and at least one cricoarytenoid unit must be preserved in order to maintain laryngeal functions properly. SCL involves the excision of both paraglottic areas, bilateral vocal cords and thyroid cartilage; further, the resection of one of the arytenoids, and possibly the pre-epiglottic space with or without the epiglottis. In STL, an extension of partial or subtotal cricoid cartilage resection is added to the classical SCL. To maintain the functional applicability of this technique, one of the cricoarytenoid units, which consists of one arytenoid cartilage, the accompanying cricoid hemi-plate, the cricoarytenoid muscle of the same side, and the superior and recurrent laryngeal nerves of the same side should be protected without risking the oncologically safe margins. The surgery includes excision of the thyroid cartilage and the entire glottic structures with the resection of subglottic structures on the tumor side. The inferior limit of the surgery extends to the first tracheal ring on the tumor side with the preservation of the non-involved hemi/partial-cricoid plate. Tracheo-hyoido-epiglottopexy (type 3a) (Figure 3) or tracheo-hyoidopexy techniques are used for reconstruction.

The indications of STL are 1) early-stage glottic tumors extending to the subglottis anteriorly or laterally, 2) glottic-subglottic

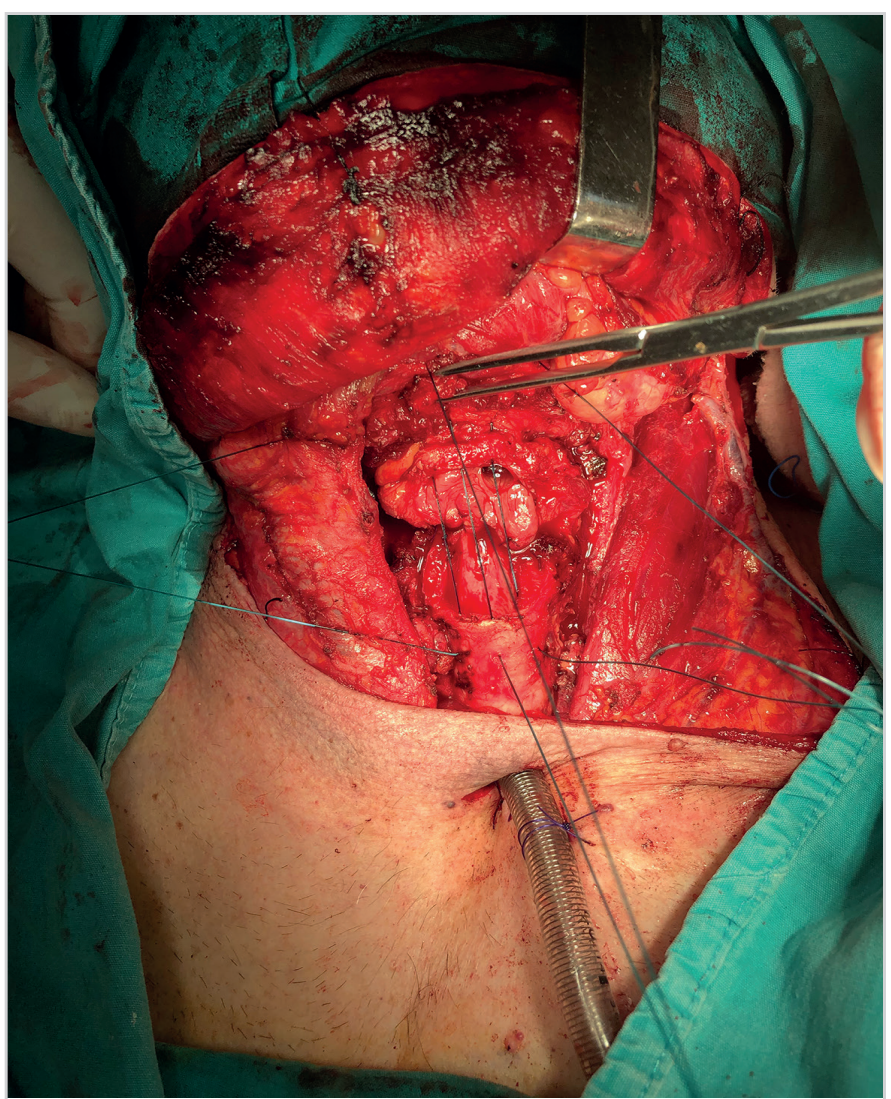

Figure 3. Surgical field image of tracheo-hyoido-epiglottopexy
T3 tumors with limited subglottic extension, 3) limited glottic subglottic T3 tumors in the paraglottic space, 4) transglottic T3 tumors extending to the paraglottic space and subglottis, 5) T4a tumors with limited anterior or lateral extralaryngeal extension.

Definite contraindications of STL are 1) advanced supraglottic tumors invading the tongue base and/or the hyoid bone, 2) advanced glottic-subglottic tumors invading the interarytenoid area or the posterior cricoid muscles, 3) massive cricoid cartilage or first tracheal cartilage invasion 4) N3 neck metastases (6). In our case series, two of our five patients were operated on for T3 tumors with subglottic extension, one for $\mathrm{T} 4 \mathrm{a}$ glottic tumor with subglottic extension and the remaining two for $\mathrm{T} 4 \mathrm{a}$ tumors with subglottic and posterior extension but no evident cricoarytenoid joint invasion. As listed in the contra-indications, in our clinic, we do not perform partial cricoid resection in the presence of major cricoid cartilage invasion. The presence of deep tumor invasion is thought to possibly decrease the oncological safety of the procedure because of the ossification of the cricoid cartilage which begins in the second decade of life. Similarly, we believe that partial resection of the cricoid is not oncologically safe in the presence of cricoarytenoid joint involvement. Therefore, in our study group, our main indication for STL was subglottic extension with cricoid perichondrium invasion.

Succo et al. (6) reported overall survival, disease-specific survival, and disease-free survival rates as $78.7 \%, 90 \%, 69.1 \%$, respectively, and locoregional and local control rates as $73.8 \%$ and $90 \%$, respectively, over a five-year period. Rizzotto et al. (7) reported their five-year overall survival and disease free survival rates as $78.9 \%$ and $68.5 \%$, respectively, and locoregional control rate as 69.6\%. Further, in the 2019 study of Garcia et al. (8), the five-year overall survival, disease-free survival, total laryngectomy-free survival and specific survival rates of STL were $82.1 \%, 83.0 \%, 80.2 \%$, and $88.2 \%$, respectively. The patients in our study group were followed with no locoregional or systemic disease. However, it is not suitable to compare our results for two reasons: firstly, the low number of patients included in our study and secondly the short follow up period, as the longest follow-up period in our study is three years.

Rizzotto et al. (7), in their study which the surgical technique of type 3 OPHL is described, state that the patients tolerated oral feeding during the postoperative first month and the nasogastric tube was removed on the $40^{\text {th }}$ day on the average. Furthermore, phonatory results were determined to be similar to the results of SCL patients at the postoperative $6^{\text {th }}$ month. In their study which they investigated the functional results of STL, Schindler et al. (9) reported that the patients were able to tolerate oral feeding with no complications and no extra supplement. Furthermore, the same study found significant detriment in the voice quality and described the characteristics of the voice as breathy and low. Lastly, the overall general quality of the patients was found satisfactory in their study. The most current article on the prognostic factors of STL was published by Succo et al. (10) in 2017, which stated that the oncological outcomes of the technique were superior to the outcomes of chemo/ra- 
diotherapy in some selected glottic/transglottic pathological T3 tumors. Similar to the literature, all of our patients started oral feeding within the first month of the surgery with no extra supplements. Four out of five patients were decannulated. Because of the low number of patients and varied follow-up periods, it would not be suitable to compare the voice results of our group with those in the literature. The two major limitations of our study are low number of patients and the short follow-up period. Since our study included only five patients and the longest follow-up period was three years, we cannot give five-year oncological results. Moreover, our patients were not evaluated with objective tests to assess phonation and swallowing. This study was planned as a case series including five patients with the aim of increasing awareness about STLs in Turkey. Further clinical studies with larger patient groups, longer follow-up periods, pre-operative and post-operative acoustic analyses and quality of life questionnaire analysis should be planned.

\section{Conclusion}

STL is an oncologically safe procedure with satisfactory functional outcomes for selected cases with advanced glottic laryngeal cancer. Awareness of the surgeons about this procedure should be increased to limit the indications of total laryngectomy.

Informed Consent: Written informed consent was obtained from the patients.

Peer-review: Externally peer-reviewed.

Author Contributions: Concept - B.B., C.D., L.A.; Design - B.B., C.D., L.A.; Supervision - B.B., C.D., L.A.; Materials - B.B., C.D., L.A.; Data Collection and/or Processing - B.B., C.D., L.A.; Analysis and/or Interpretation - B.B., C.D., L.A.; Literature Search - B.B., C.D., L.A.; Writing - B.B., C.D., L.A.; Critical Reviews - B.B., C.D., L.A.

Conflict of Interest: The authors have no conflicts of interest to declare.
Financial Disclosure: The authors declared that this study has received no financial support.

\section{References}

1. Majer H, Reider W. [Technic of laryngectomy permitting the conservation of respiratory permeability (cricohyoidopexy)]. Ann Otolaryngol Chir Cervicofac 1959; 76: 677-83.

2. Laccourreye H, Ménard M, Fabre A, Brasnu D, Janot F. [Partial supracricoid laryngectomy. Technics, indications and results.] Ann Otolaryngol Chir Cervicofac 1987; 104: 163-73.

3. Laccourreye O, Brasnu D, Jouffre V, Couloigner V, Naudo P, Laccourreye H. [Supra-cricoid partial laryngectomy extended to the anterior arch of the cricoid with tracheo-crico-hyoido-epiglottopexy. Oncologic and functional results.] Ann Otolaryngol Chir Cervicofac 1996; 113: 15-9.

4. Rizzotto G, Succo G, Lucioni M, Pazzaia T. Subtotal laryngectomy with tracheohyoidopexy: a possible alternative to total laryngectomy. Laryngoscope 2006; 116: 1907-17. [Crossref]

5. Succo G, Peretti G, Piazza C, Remacle M, Eckel HE, Chevalier $\mathrm{D}$, et al. Open partial horizontal laryngectomies: a proposal for classification by the working committee on nomenclature of the European Laryngological Society. Eur Arch Otorhinolaryngol 2014; 271: 2489-96. [Crossref]

6. Succo G, Bussi M, Presutti L, Cirillo S, Crosetti E, Bertolin A, et al. Supratracheal laryngectomy: current indications and contraindications. Acta Otorhinolaryngol Ital 2015; 35: 146-56.

7. Rizzotto G, Crosetti E, Lucioni M, Bertolin A, Monticone V, Sprio AE, et al. Oncologic outcomes of supratracheal laryngectomy: critical analysis. Head Neck 2015; 37: 1417-24. [Crossref]

8. Garcia AM, Dias FL, Gonçalves AJ, Cernea CR, Freitas EQ, Menezes MB, et al. Supratracheal laryngectomy: a multi-institutional study. Braz J Otorhinolaryngol 2019; S1808-8694: 307031.

9. Schindler A, Fantini M, Pizzorni N, Crosetti E, Mozzanica F, Bertolin A, et al. Swallowing, voice, and quality of life after supratracheal laryngectomy: Preliminary long-term results. Head Neck 2015; 37: 557-66. [Crossref]

10. Succo G, Fantini M, Rizzotto G. Supratracheal partial laryngectomy: indications, oncologic and functional results. Curr Opin Otolaryngol Head Neck Surg 2017; 25: 127-32. [Crossref] 